Convergences francophones 6.2 (2020): 74-82

http://mrujs.mtroyal.ca/index.php/cf/index

\title{
De l'approche par compétences à l'approche par compétence : les réformes du curriculum de français en Tanzanie
}

\author{
Alfred Mulinda \\ Université de Calgary
}

En 2005, le Ministère tanzanien de l'Éducation a entrepris une réforme qui l'a conduit à introduire un curriculum d'enseignement axé sur l'approche par compétence (competence based curriculum) pour remplacer le curriculum d'enseignement axé sur le contenu (content based curriculum) (Paulo \& Tilya 2014). Suite à ces directives, les nouveaux plans de cours ainsi que les nouveaux manuels d'enseignement seront conçus pour s'aligner sur les exigences du nouveau curriculum. Le présent travail est une analyse de deux manuels d'enseignement du français en Tanzanie, à savoir, Transafrique (Godard et al. 1991), utilisé avant les changements, et On y va! (Mazauric \& Sirejols 2002), proposé ultérieurement.

Dans le cadre de cette analyse, nous partirons du constat que le curriculum d'enseignement axé sur l'approche par compétence viserait, dans le cas de l'enseignement des langues, la compétence de communication. Comme nous allons le voir en détail par la suite, la compétence de communication est un élément cible de l'approche par compétence. Notre analyse de Transafrique et On y va! aura donc pour but de découvrir les affiliations méthodologiques des deux manuels. Notre objectif principal est de découvrir si On y va! s'inspire de l'approche par compétence, ce qui nous permettra de savoir finalement si les changements curriculaires proposés se reflètent également dans le choix des méthodes d'enseignement. Nous avons également voulu savoir si Transafrique ne pourrait pas répondre lui-aussi aux exigences du nouveau curriculum.

\section{Présentation générale des manuels}

\section{Transafrique}

Transafrique est un manuel de français conçu pour le secondaire en Afrique anglophone de l'Ouest. Le manuel compte trois séries, à savoir Transafrique 1, 2 et 3. Les séries s'adressent aux élèves des trois premières années du premier cycle du secondaire. La première série, qui sera l'objet de notre analyse, compte 96 pages. Comme stipule clairement son introduction, le manuel vise à donner la base de la langue française du quotidien tant à l'oral qu'à l'écrit aux adolescents de 12 à 13 ans. Le but est de permettre à ces derniers de se servir de la langue française dans la classe comme en dehors de la classe. Tous les thèmes vus dans cette série de Transafrique 1 se rapportent à la vie quotidienne, et la plupart des dialogues sont situés dans le contexte anglophone africain. Les actes de parole à travailler sont clairement présentés dans la table des matières.

Transafrique 1 propose 12 unités, et chacune contient 3 parties : 
La première partie s'intitule Oral. Cette partie introduit les activités de compréhension et de production orales. Ces activités présentent les actes de parole et les structures à travailler dans l'unité. A partir des images, l'enseignant dit ce qui se passe et les apprenants écoutent. Ensuite, il leur pose des questions de compréhension. L'enseignant finit par engager les apprenants dans plusieurs activités de prise de parole. Ces activités impliquent l'apprenant et l'enseignant ainsi que les apprenants entre eux.

La deuxième partie, Lecture, consiste d'un texte sous la forme d'une bande dessinée dialoguée contenant les structures présentées dans la partie oral précédente. Pour commencer, l'enseignant lit le dialogue tandis que les apprenants le suivent. Ensuite, les apprenants lisent le dialogue eux-mêmes et répondent aux questions. Après la lecture, l'enseignant guide les apprenants à bien prononcer certains mots dans la partie Orthographe et prononciation. Puis, la section présente le vocabulaire et le point de grammaire présentés dans l'unité.

Enfin, la partie Exercices propose des exercices de révision des points de grammaire ainsi que des actes de parole travaillés au sein de l'unité. Ces exercices restent majoritairement au niveau des phrases courtes individuelles, écrites ou orales. On note parfois la présence de travaux en paires ou en groupes ainsi que des jeux de rôle. La section exercices se clôt avec soit la section l'arbre aux poèmes (poème impliquant les structures et le lexique vus dans l'unité), soit la section fais le point (activité guidant l'apprenant à travailler certains actes de parole vus dans l'unité). Tous les mots utilisés dans les poèmes se trouvent dans le glossaire.

\section{Ony va!}

De même que Transafrique, On y va! est conçu pour le secondaire en Afrique anglophone de l'Ouest. Le manuel compte trois volumes, à savoir On y va 1, 2 et 3 , qui s'adressent aux élèves du premier cycle du secondaire. Le premier volume, qui sera objet de notre analyse, compte 96 pages. Les instructions et les explications de grammaire sont données en anglais. Les titres des sections sont en français. Ce volume propose 12 unités autour des actes de parole et des points de grammaire. Toutes les trois unités, le manuel propose deux pages d'exercices, intitulées Etapes, couvrant le contenu grammatical travaillé dans les trois unités. Chaque unité est composée comme suit :

A la première page, On y va! propose une bande dessinée dialoguée avec les personnages-héros du manuel, qui sont de jeunes africains francophones. Chaque unité reprend en son titre les mots clés du dialogue en question. Le dialogue sert à introduire les actes de parole et les points de grammaire à développer tout au long des étapes ultérieures. A la deuxième page, On y va! contient des activités qui guident l'apprenant à se servir dans des phrases des actes de parole et des points de grammaire présentés dans le dialogue précédent. Ces activités proposent trois actes de parole à travailler et ont pour instructions look, think and repeat, et Your turn: talk to a partner. Cette page contient également le vocabulaire accompagnant les actes de parole développés dans l'unité. Cette section est intitulée Partners. La 
troisième page intitulée Lire et comprendre contient des documents authentiques écrits, suivis de questions de compréhension qui évoquent toujours les actes de parole et le point de grammaire travaillés au sein de l'unité. La quatrième page, intitulée Apprendre et appliquer, propose des activités qui situent le point de grammaire travaillé et finit par l'expliciter.

La cinquième page a deux sections. La première section, intitulée sounds, music, propose des activités qui permettent à l'apprenant de découvrir et de pratiquer les différents sons et intonations du français à partir de documents sonores. La deuxième section, intitulée role play, propose à l'apprenant des jeux de rôles simulant les activités de la vie quotidienne provenant des actes de parole travaillés dans l'unité. Ces activités proposent à l'apprenant de travailler en pair ou en groupe. La dernière page de chaque unité propose à l'apprenant des activités écrites se rapportant au thème de l'unité. A la fin du livre, le manuel propose un résumé des points de grammaire. On y va! est aussi accompagnée du cahier d'exercices.

\section{La méthodologie utilisée par les auteurs}

Un examen attentif de l'introduction de Transafrique 1 et de la partie Organization of each unit d'On y va!1, du contenu de leurs unités, ainsi que de la manière et la fréquence dont les activités ont été conçues et présentées, suggère que les auteurs se sont principalement appuyés sur les principes de l'approche par compétence (ou approche communicative comme nous le verrons par la suite) pour concevoir les manuels. Avant d'illustrer l'affiliation des deux manuels aux approches communicatives, il est essentiel de découvrir brièvement à quoi renvoient ces approches.

\section{L'approche par compétence}

L'approche par compétence compte parmi les nombreuses appellations méthodologiques renvoyant aux pratiques d'enseignement des langues visant la compétence de communication. Elle fait partie des approches communicatives, terme général se référant à l'ensemble de ce type de pratique d'enseignement. Nous nous servirons ici de façon interchangeable des termes approche par compétence, approche communicative ou bien approches communicatives (au pluriel) car il s'agit de toute approche/procédé en classe qui aura pour cible la compétence de communication en s'inscrivant dans les principes détaillés ci-dessous. Certains auteurs, dont Cuq (2003) se servent de ces appellations de pareille façon (au singulier comme au pluriel).

Les approches communicatives (voir Cuq 2003, Cuq \& Gruca 2005, Richards 2006, Richards \& Rodgers 2014) renvoient aux dispositifs de choix méthodologique visant à développer chez l'apprenant la compétence à communiquer (ou compétence de communication). Pour ce faire, l'enseignant propose à l'apprenant un cours de langue autour d'objectifs de communication. Le but est de permettre à l'apprenant d'acquérir une compétence de communication en langue seconde. Pour 
avoir ladite compétence, l'enseignant propose à l'apprenant un cours centré sur des actes de parole en utilisant des documents authentiques et des activités d'expression comme les simulations et les jeux de rôle ou des activités de compréhension qui imitent les situations de communication de la vie réelle.

Dans un cours de langue s'inspirant des approches communicatives, les règles de grammaire sont importantes mais ne constituent pas le but principal de l'apprentissage. Elles sont découvertes ultérieurement et de façon implicite lors de la maîtrise des actes de parole (lors des tentatives de maîtrise de la communication). Autrement dit, comme le souligne Germain (1991), l'apprenant apprend la langue seconde en communiquant dans cette langue. ${ }^{1}$ Dans ce genre de cours, le rôle de l'enseignant est celui d'organisateur et de facilitateur des activités communicatives au sein de la classe et non pas de détenteur de savoir dont le rôle serait de passer les savoirs aux apprenants (Richards \& Rodgers 2014, Richards 2006). L'objectif des approches communicatives étant la compétence de communication, il s'oppose à celui des anciennes méthodologies non-communicatives dont le but était la maîtrise de la compétence grammaticale (voir aussi Brumfit 1979).

Il faut préciser, comme remarquent Richards (2006) ainsi que Richards \& Rodgers (2014), que les approches communicatives ne disposent pas de procédures typiques à suivre pour dispenser le cours de langue. Ainsi, toute manœuvre en classe de langue visant la compétence de communication à travers les situations authentiques de la vie quotidienne et visant les besoins communicatifs ultérieurs des apprenants est considérée comme une approche communicative.

\section{Caractéristiques inscrivant On $y$ va! et Transafrique dans l'approche communicative}

Afin de déterminer dans quelle mesure ces manuels s'inscrivent dans l'approche communicative, nous allons procéder à l'observation de l'introduction et de la page de couverture de Transafrique, ${ }^{2}$ ainsi que des unités des deux manuels. Nous porterons en particulier notre attention sur certains éléments tirés de la grille d'analyse des manuels (Cuq \& Gruca 2005): présentation des tables des matières, place donnée à la compétence de communication versus la compétence linguistique, la place de l'audiovisuel et du document authentique ainsi que l'inventaire de types d'exercices proposés.

\section{Présentation des tables des matières}

Transafrique propose une table des matières qui compte sept colonnes intitulées Unité, Notions/Actes de parole, Oral, Lecture, Orthographe et prononciation, Grammaire/Conjugaisons et Vocabulaire. La deuxième colonne, Notions/Actes de

\footnotetext{
${ }^{1}$ L'apprenant est considéré comme étant un « communicateur » car partenaire dans la négociation du sens ou du message communiqué (Germain Le point 206).

${ }^{2}$ On y va! ne dispose pas d'introduction, mais la partie Organization of each unit dans les premières pages fournit quelques détails introductoires au manuel.
} 
parole, articule explicitement un jargon appartenant aux approches communicatives. Le concept actes de parole est l'un des concepts clés des approches communicatives. Comme nous avons indiqué ci-haut, la visée principale de ces approches est de permettre à l'apprenant d'une langue seconde de maîtriser les actes de paroles de la langue cible.

Selon l'approche communicative, pour qu'un locuteur de langue seconde arrive à se servir des actes de paroles, il lui faut une situation de communication en rapport avec ces actes. Pour ce faire, Transafrique propose une colonne appelée Lecture, consacrée aux situations où les actes de parole en question peuvent se produire. Par exemple, pour l'acte de parole Stating/asking likes and dislikes, Transafrique propose dans ecture une situation de communication à la buvette, comme l'endroit où la conversation va se passer. Une autre chose propre à l'approche communicative dans Transafrique 1 est le fait que la grammaire n'est pas explicitée dès le début. Comme nous avons vu ci-dessus, dans un cours s'inspirant de l'approche communicative, les règles de grammaire (la compétence linguistique) arrivent par la suite pour appuyer la maîtrise des actes de parole,

Quant au manuel On $y v a !$, la table des matières indique clairement que le manuel s'inscrit aussi dans l'approche communicative. Dans la table des matières, qui compte très peu de contenu, la section Communication vient avant Grammar. Cette section propose les actes de parole visés pour un cours en question. Après que les actes de parole sont proposés, la colonne Grammar liste les points de grammaire qui vont faciliter la pratique des actes de parole proposés. Cela montre que le but principal du manuel est que les apprenants puissent maitriser les actes de parole en question, et que pour ce faire, ils ont besoin des règles de grammaire. Cela montre que la grammaire pour On y va! n'est pas le but principal de l'enseignement des langues secondes, mais un moyen d'arriver à la maîtrise des actes de parole, de la compétence de communication.

\section{communication}

Place accordée à la compétence linguistique vs la compétence de

Comme nous l'avons indiqué plus haut, le but principal des approches communicatives est la compétence de communication. Pour s'approprier la compétence de communication, l'apprenant doit pouvoir maîtriser les actes de paroles qui se rapportent à une situation de communication impliquant la langue quotidienne. Les deux manuels donnent tous deux une place prioritaire à la réalisation des actes de parole avant d'entamer la grammaire de façon explicite. A regarder les pages d'On y va!, par exemple, nous voyons clairement que l'unité commence par un dialogue à la première page. Le dialogue sert à introduire les actes de parole et, de façon implicite, des points de grammaire à développer tout au long des étapes ultérieures.

La deuxième page contient des activités qui guident l'apprenant à se servir dans des phrases des actes de parole et des points de grammaire présentés implicitement dans le dialogue précédent. Ces activités proposent trois actes de parole à travailler et 
ont comme instructions look and repeat, talk to a partner, look think and repeat. La troisième page intitulée lire et comprendre contient un document authentique écrit, suivi par des questions de compréhension qui évoquent toujours les actes de parole et le point de grammaire travaillé au sein de l'unité. C'est en bas de la quatrième page, intitulée apprendre et appliquer que le point de grammaire est explicité, et très brièvement. Après l'explicitation du point de grammaire, les deux pages suivantes proposent toujours des activités relevant de l'emploi de la langue quotidienne, y compris dans des travaux par groupe, par deux ainsi que des simulations qui imitent les activités sociales de la langue.

Dans le cas de Transafrique, nous remarquons une tendance quasiment identique au regard de la primauté de la compétence de communication vis-à-vis de la compétence linguistique. Selon son introduction et sa page de couverture, Transafrique se définit comme une méthode communicative visant à donner les bases de la langue française du quotidien tant à l'oral qu'à l'écrit pour permettre aux apprenants de se servir de la langue française tant dans la classe que dans la vie quotidienne. Tous les thèmes vus dans ce niveau de Transafrique se rapportent à la vie quotidienne et la plupart des dialogues sont situés dans le contexte anglophone africain. Les thèmes, appelés actes de parole, montrent clairement que les auteurs avaient déjà en tête les principes de l'approche communicative. Dès le début de l'unité de Transafrique, le but est de travailler les actes de parole qui se présentent tout premièrement dans la partie orale sous la forme de dessins qui décrivent un contexte de communication, suivi par des questions de compréhension. Après cette partie, les activités suivantes invitent l'apprenant à se servir des actes de parole dans des phrases et à partir des dialogues. La règle de grammaire est explicitée à la quatrième page. Ce procédé nous fait conclure que ce manuel relève des principes de l'approche communicative.

\section{Place de l'audiovisuel et du document authentique}

Les seuls éléments visuels dans Transafrique figurent dans des images placées notamment aux première et troisième page de chaque unité, ainsi que dans la section qui présente le vocabulaire. A la première page, la première activité invite l'apprenant à regarder les images alors que l'enseignant les décrit. Ce faisant, l'apprenant parvient à tirer du vocabulaire à partir de l'image et finit en répondant aux questions associées. A la troisième page des unités, il y a une bande dessinée dialoguée qui sert aussi à faciliter la saisie rapide du contexte de communication par l'apprenant. Certaines sections présentant le vocabulaire se servent également des images. Il y a des images dessinées en couleur et il n'y a pas de documents authentiques. Aussi, est-il que, à part la voix de l'enseignant qui est le seul modèle de prononciation et d'intonation, le manuel ne dispose pas de documents sonores. Toutefois, l'utilisation du visuel ainsi que les bandes dessinées dialoguées permet à l'apprenant de se familiariser avec les actes de parole véhiculés par ces activités, faisant de Transafrique un manuel principalement communicatif. 
Quant à On y va!, la troisième page de chaque unité, intitulée lire et comprendre, propose un document authentique écrit, à partir duquel l'apprenant répond aux questions de compréhension. En ce qui concerne l'audio, la section sounds, music, comme nous l'avons indiqué plus haut, propose des activités Mis à part la troisième et la quatrième des six pages de chaque unité, les activités que propose On y va! sont largement accompagnées d'images en couleur, ce qui n'est pas le cas pour Transafrique. Cette différence peut être attribuée au décalage entre les années de publication de ces méthodes. L'accès éventuel de l'apprenant à la compétence de communication ne serait en aucun cas entravé par la couleur des dessins.

\section{Inventaire de types d'exercices proposés}

Les deux manuels proposent une variété d'exercices pour s'assurer que l'apprenant maîtrise les actes de parole retenus. Même si Transafrique se réclame de l'approche communicative, et qu'il propose une variété d'exercices permettant à l'apprenant de retravailler les actes de parole et le point de grammaire proposés dans chaque unité, il faut remarquer que la plupart de ses exercices posent la pratique de la règle de grammaire. Outre la proposition des actes de parole et les activités qui les impliquent, la plupart des activités qui impliquent la prise de parole se limitent au niveau de la phrase et des échanges de phrases courtes parmi les apprenants. $\mathrm{Au}$ sein de l'unité, par exemple, il y a seulement deux activités de jeu de rôle, l'un des types d'activités recommandées par l'approche communicative.

Si Transafrique propose plus d'exercices de grammaire au sein de ses unités que d'activités communicatives, cela ne sera pas le cas pour On y va!. A l'exception des exercices de la section Etapes, exercices complémentaires qui résument les unités précédentes et proposent la pratique de la règle de grammaire, le reste des activités proposées par le manuel invite l'apprenant à pratiquer la langue de tous les jours, comme par exemple : Talk about your timetable, Tell your partner what you do every day, Say what the weather is like, Talk about your activities during different months of the year,

En ce qui concerne le nombre élevé des exercices de grammaire, nous pouvons attribuer cette tendance au fait que Transafrique a été conçu au début des années 1990, époque où l'approche communicative commençait à révolutionner la didactique des langues en se révoltant contre les méthodologies noncommunicatives tout en constituant cependant l'amélioration ou le continuum des principes de des premières (Amedegnato 2010) : ceci expliquerait la permanence de certains éléments saillants des méthodologies précédentes même dans les manuels d'orientation communicative.

\section{Conclusion \& perspectives}

Les manuels Transafrique et On y va! s'inscrivent donc principalement dans l'approche communicative car leur philosophie de cours de langue les achemine vers la compétence de communication. En effet, ces manuels ont pour but la 
réalisation des actes de parole spécifiques à l'apprenant et présentent d'abord la langue en situation avant de présenter la règle de grammaire de façon implicite. Nous remarquons aussi que l'enseignement du français en Tanzanie s'inscrivait déjà dans l'approche communicative avant même 2005, à travers l'utilisation de Transafrique. Il faut tout de même remarquer que le programme sur lequel devait s'appuyer Transafrique était toujours paradoxalement non-communicatif.

Toutefois, il existe quelques décalages entre ces méthodes dans la façon dont elles procèdent vers la réalisation des actes de parole. Par exemple, Transafrique manque de documents sonores, explicite un peu plus la règle de grammaire dans ses activités, et le manuel manque d'images en couleur, entre autres choses. Ceci n'exclut pas pour autant ce manuel du champ communicatif. Nous pouvons attribuer cette différence aux méthodes de production de manuels de FLE ou FLS selon les époques. L'image, par exemple, s'est améliorée dans le temps, raison pour laquelle il est logique de trouver une plus grande variété de couleurs dans des méthodes d'aujourd'hui que dans celles d'autrefois. Si l'image en couleur augmente l'effet mnémonique chez l'apprenant, son manque ne réduit pas le manuel à un manuel relevant du champ non-communicatif.

Enfin, même si cela va sans dire que le nouveau manuel correspond aux nouvelles réformes du curriculum, dans la mesure où il est conforme aux principes des approches communicatives, il ne remplace pas obligatoirement le précédent, car ils sont tous pertinents pour le nouveau syllabus. Malgré le fait que On y va! utilise plus de technologie que Transafrique, les deux manuels peuvent être tous deux utilisés dans le cadre de la réforme de 2005. Reste que l'actualité des contenus demande peut-être à être revue dans la méthode la plus ancienne, voire même dans celle de 2002. La mise à jour des méthodes de FLE ou FLS est toutefois une question qui excède les limites de notre propos. 


\section{Bibliographie}

Amedegnato, Ozouf, S. « Méthodologies d'enseignement des langues : les leçons de l'histoire. \Enjeux linguistiques et sociaux au Canada en devenir: Identité, diversité et pratiques pédagogiques, Dirigé par Sylvie Roy et Chantal Berlinguette Calgary : Blitzprint, 2010.

Brumfit, Christopher J., \& Keith Johnson, directeurs. The communicative Approach to Language Teaching. Oxford: Oxford University Press, 1979.

Cuq, Jean-Pierre. Dictionnaire de didactique du français. Paris: CLE international, 2003.

Cuq, Jean-Pierre et Isabelle Gruca. Cours de didactique du français langue étrangère et seconde. Grenoble : Presses universitaires de Grenoble, 2005.

Germain, Claude. Évolution de l'enseignement des langues : 5000 ans d'histoire. Paris : Clé International, 1993.

---. Le point sur l'approche communicative en didactique des langues. Montréal : Centre éducatif et culturel, 1991.

Godard, Roger, Alain Pacthod et Chantal Paisant. Transafrique 1. Vanves : Hachette, 1991.

Mazauric, Catherine et Evelyne Sirejols. On y va! : Méthode de Français. Paris : CLE International, 2002.

Paulo, Albert et Frank Tilya. "The 2005 secondary school curriculum reforms in Tanzania:Disjunction between policy and practice in its implementation." Journal of Education and Practice, vol. 5, no. 35, 2014, pp. 114-122.

Richards, Jack C. Communicative Language Teaching Today. New York: Cambridge University Press, 2006.

Richards, Jack C., et Theodore S. Rodgers. Approaches and Methods in Language Teaching. 3e édition. New York: Cambridge University Press, 2014. 